\title{
A Review on Bandwidth Improvement using Metamaterial
}

\author{
Miss. Varsha Chavan', Mr. Uday Patil ${ }^{2}$ \\ ${ }^{1}$ Student, ${ }^{2}$ Assistant Professer \\ Electronics Technology, Department of Technology, Shivaji University, Kolhapur, Maharashtra, India
}

\begin{abstract}
Over the past one decade, there is a rapid growth in development of various applications involving wireless communication. The performance of all such wireless systems depends on the design and proper functioning of the antenna. Metamaterials are artificial metallic structures having simultaneously negative permittivity $(\varepsilon)$ and permeability $(\mu)$, which leads to negative refractive index. No other material in the world shows the above properties like Metamaterial. Due to these unusual properties Metamaterial can change the electric and magnetic property of electromagnetic wave passing through it and because of these reasons when Metamaterial is used in the fabrication of antennas the required properties can be enhanced. Using this Metamaterial antenna the demerits of ordinary patch antenna like narrow bandwidth can be overcome.
\end{abstract}

\section{KEYWORDS: Metamaterial, WLAN}

\section{INTRODUCTION}

The word "Meta" is taken from Greek whose meaning is "beyond". The first contribution to this topic was made in 1968 by V. G. vasalago. He said that materials with both negative permittivity $(\varepsilon)$ and negative permeability $(\mu)$ are theoretically possible [1] after that in 1999 john pendry identify a practical way and proposed design of periodically arranged Thin-Wire (TW) structure that depictsthe negative value of effective permittivity [2].Later, then in 2000 smith discovered a new LHM(left handed material)that shows simultaneously negative permittivity and permeability and carried out microwave experiments to test its uncommon properties.[3]

\section{Classification of metamaterial:}

Classification of metamaterial depend upon permittivity $(\varepsilon)$ and permeability $(\mu)$ of structure. On the basis of permittivity $\varepsilon$ and permeability $\mu$, the metamaterials are classified in following four groups as shown in Fig. 1:

\begin{tabular}{c|c}
$\begin{array}{c}\text { ENG material } \\
(\varepsilon<0, \mu>0) \\
\text { Plasmas }\end{array}$ & $\begin{array}{c}\text { DPS material } \\
(\varepsilon>0, \mu>0) \\
\text { Dielectrics }\end{array}$ \\
\hline $\begin{array}{c}\text { DNG material } \\
(\varepsilon<0, \mu<0)\end{array}$ & $\begin{array}{c}\text { MNG material } \\
(\varepsilon>0, \mu<0) \\
\text { Not found in nature }\end{array}$ \\
& $\begin{array}{c}\text { Gyrotropic } \\
\text { magnetic materials }\end{array}$ \\
& 0 Zero-index
\end{tabular}

A. Double Positive (DPS) Material The materials which have both permittivity \& permeability greater than zero $(\varepsilon>$ $0, \mu>0$ ) are called as double positive (DPS) materials. Most occurring media (e.g. dielectrics) fall under this designation.
B. Epsilon Negative (ENG) Material If

a material has permittivity less than zero and permeability greater than zero $(\varepsilon<0, \mu>0)$ it is called as epsilon negative (ENG) material In certain frequency regimes , many plasmas exhibit these characteristics.

\section{Mu Negative (MNG)}

MaterialIf a material has permittivity greater than zero \& permeability less than zero $(\varepsilon>0, \mu<0)$ it is called as mu negative (MNG)material. In certain frequency regimes, some gyro tropic material exhibits these characteristics

\section{Double Negative}

(DNG) Material If a material has permittivity \& permeability less than zero $(\varepsilon<0, \mu<0)$ it is termed as double negative (DNG) material.This class of materials can only been produced artificially.

\section{LITERATURE SURVEY}

There are different techniques can be used in metamaterial,

Deschamps first proposed the concept of the MSA in 1953 [4]. However, practical antennas were developed by Munson and Howell in the 1970s.[5,6] With increasing requirements for personal and mobile communications, the demand for smaller and low-profile antennas has brought the MSA to the forefront. Increasing the BW of MSAs has been the major thrust of research in this field[4]. Design of micro strip patch antenna with bandwidth improvement is popular field for researchers. The literature review is, Bandwidth Enhanced Micro strip Patch Antenna Using Metamaterials

Bashir D. Bala1, Mohamad Kamal A. Rahim2, N.A. Murad3 M. F. Ismail4, H.A.Majid5 Introduced a metamaterial antenna with triangular resonator and thin wire. The antenna is excited through a single unit cell left handed material (LHM). The results of the antenna shows that $58 \%$ of bandwidth is achieved with a return loss of $10 \mathrm{db}$,the maximum gain of antenna is 4.45

Bashir D. Bala1, Mohamad Kamal A. Rahim2, N. A. Murad3 In this paper, a metamaterial antenna is based on Split Ring Resonator (SRR) and a Thin Wire (TW) is proposed and 
studied. The antenna consists of six unit cells and a thin wire of Left Handed Materials (LHM) arranged in 2x3 printed on same side of substrate. For connection of the LHM's to the radiating edge of a microstrip patch antenna TW stub of the unit cell is used. By using these techniques, the bandwidth of the metamaterial antenna is twice that of the ordinary patch antenna at the same operating frequency.

Mostafa1.M.Bakry2, Adel B. Abdel-Rahman3, Hesham F. A. Hamed 4 proposed a antenna with a periodic structure of Complementary G-Shape Split Ring Resonator (CGSRR) to improve the gain of microstrip patch antenna (MPA). GShape Split Ring Resonator (CGSRR) work as a left-handed material (LHM) with negative permittivity and permeability in the frequency band of operation. Antenna is designed using co-axial probe feed. Array of CGSRRs are loaded around the rectangular patch to improve the gain and bandwidth of the MPA. CGSRRs around the patch antenna improves the gain by $2.5 \mathrm{~dB}$ over the original values of the total gain of the conventional antenna and also increases the fractional bandwidth of the antenna from $3.5 \%$ to $5.1 \%$.

Mr. Chaitanya Vijaykumar Mahamuni is proposed a microstrip patch antenna. In this paper the conventional microstrip antenna is used with metamaterial cover.atch antennas have various capabilities so that these factors or capabilities, patch antennas are able to overcome the rising demands in the field of wireless communication like high gain, high power, maximized throughput and low losses. The stacking of patch antennas i.e. the use of one patch over other is an effective method to improve their performance for the dual frequency and broadband operation which is required in most of the high frequency wireless applications. The gap between microstrip patch antenna and cover subjected to an excitation will lead to a strong mutual coupling between them. This is the reason for the bandwidth enhancement. The results are shown by using matlab.

Aadya Pant1, R. P. S. Gangwar2 proposed antenna with improvement in bandwidth. In this paper tworectangular paracitic stubs are adeed near the feeding line. Two semicircular slots in the lower edge of the ground plane are introduced along with the dual split ring resonator (SRR) which is displaced from the centre for bandwidth enhancement. Due to the arrangement the bandwidth of antenna is increased by $158 \%$ ranging from $2.28-18.9 \mathrm{GHz}$. The size of the proposed antenna is $37^{*} 37^{*} 0.8 \mathrm{~mm} 3$.

Yoon jae Lee1, Simon Tse2, Yang Hao3, and Clive G. Parini4 .In this paper bandwidth of the microstrip patch antenna is improved by using a special approach is to use a high permittivity dielectric substrate in order to decrease the guided wavelength, and hence the overall antenna size. In this paper, they propose a new design approach to the realization of compact antennas with improved impedance bandwidth using a ground plane loaded with complem entary split-ring resonators (CSRRs). They examine the resonant frequency, impedance bandwidth, and radiation characteristics of antenna. The experimental results demonstrated that significant size reduction is possible for a microstrip antenna without sacrificing the bandwidth by using the CSRR loaded ground plane. The fabricated antenna achieves a $69 \%$ reduction in the resonant frequency as well as $67 \%$ improvement in the bandwidth compared to the conventional antenna.
Farzad Alizadeh1, Changiz Ghobadi2, Javad Nourinia3, Rasoul Zayer4 are presents a broadband, high efficiency patch antenna that utilizes a novel complementary split ring resonators(CSRRs) embedded on the ground plane. The CSRR in ground plane results a beneficial improvement in impedance bandwidth, efficiency, compactness and directivity in comparison to a conventional microstrip antenna. Feeding technique used is coaxial-feed . the microstrip patch antenna integrated with a simple CSRRs structure that is cut from the ground plane. Inserting two crossovers with via bridges to the CSRRs structure facilitates a nearly complete impedance match to the source over a wider bandwidth and also maintain a high, $89 \%$, radiation efficiency. The designs and their performance characteristics were simulated with the High Frequency Structure Simulator (HFSS) and verified using the time domain CST Microwave studio simulator and Sonnet EM software. Test results of the fabricated antennas show a good correlation with simulated outcomes.

Chirag Arora 1, Shyam S. Pattnaik 2, and Rudra N. Baral3 This paper presents a microstrip fed patch antenna array, loaded with metamaterial superstrate. An unloaded antenna array resonates at IEEE 802.16a 5.8 GHz Wi-MAX band with gain of $4.3 \mathrm{dBi}$ and bandwidth of $425 \mathrm{MHz}$. in this paper same array loaded with metamaterial superstrate having layer of split ring resonators gain and bandwidth improved 8 $\mathrm{dBi}$ and $680 \mathrm{MHz}$ respectively. $86 \%$ gain and $60 \%$ bandwidth enhancement achieved.

\section{CONCLUSION}

This review paper shows techniques and methods for enhancing the bandwidth of MPA using metamaterial. Metamaterials are Metamaterials is a new term describing a concept of artificial, man-made materials composed of small cells containing at least two different natural materials. Metamaterials can exhibit much more pronounced electromagnetic properties than natural materials so they can be used in the new high-speed broadband systems. However recent studies and experiment found that modification of shape of split ring resonator, by using array of resonators with thin wire. Also by stacking of patch antennas,by introducing split ring resonator at the ground plane.

\section{REFERENCES}

[1] V. G. Veselago, "The electrodynamics of substances with simultaneously negative values of " $\varepsilon$, and $\mu$ ", Sov. Phys.Usp,Vol. 47, pp.509-514, Jan.-Feb, 1968.

[2] J. P. Pendry, A. J. Holden, D.J. Robbins, W. J. Stewart, IEEE. Trans. Microwave, Theory Technol. 47, 2075 1999.

[3] D.R. Smith,W.J. Padila, D.C. Vier, S.C. Nemat- Nasser, S. Schultz, Phys.Rev. Lett. 84, 4184,2000

[4] Des champs, G. A., "Microstrip Microwave Antennas," Proc. 3rd USAF Symposium on Antennas, 1953.

[5] Munson, R. E., "Single Slot Cavity Antennas Assembly," U.S. Patent No. 3713162, January 23, 1973.

[6] Munson, R. E., "Conformal Microstrip Antennas and Microstrip Phased Arrays," IEEE Trans. Antennas Propagation, Vol. AP- 22, 1974, pp. 74-78.

[7] Design and analysis of metamaterial antenna using Triangular resonator Bashir D. Bala1, Mohamad Kamal 
A. Rahim2, N.A. Murad3 M.F.Ismail4 , H.A.Majid5 IEEE IEEE TRANSACTIONS ON ANTENNAS AND PROPAGATION, VOL. 48, NO. 8, AUGUST 2012

[8] Bandwidth Enhanced Microstrip Patch Antenna Using Metamaterials Bashir D. Bala1, Mohamad Kamal A. Rahim2, N. A. Murad3 2012 IEEE Asia-Pacific Conference on Applied Electromagnetics (APACE 2012), December 11 - 13, 2012, Melaka, Malaysia

[9] Gain and Bandwidth Improvement of Microstrip Patch Antenna using Comple- mentary G-shape Split Ring Resonator 1Mostafa. M.Bakry, 2Adel B. Abdel-Rahman, 3Hesham F. A. Hamed 31st National Radio Science Conference (NRSC2014) April 28 - 30, 2014,

[10] Performance Enhancement of Microstrip Patch Antenna using Metamaterial Cover Mr. Chaitanya Vijaykumar Mahamuni International Conference on Global Trends in Signal Processing IEEE 2016
[11] Performance improvement of a slot antenna using SRR and modified ground plane Aadya Pant, R. P. S. Gangwar 978-1-5090-3480-2/16/\$31.00 (C)2016 IEEE

[12] A Compact Microstrip Antenna with Improved Bandwidth Using Complementary Split-Ring Resonator (CSRR) Loading Yoonjae Lee, Simon Tse, Yang Hao, and Clive G. Parini 1-4244-0878-4/07/\$20.00 C2007 IEEE

[13] Bandwidth Enhancement of Patch Antennas Loaded With Complementary Split-Ring Resonators Farzad Alizadeh, Changiz Ghobadi, Javad Nourinia, Rasoul Zayer $20147^{\text {th }}$ International Symposium on Telecommu-nications (IST'2014) 978-1 4799-53592/14/\$31.00 @2014 IEEE

[14] SRR Superstrate for Gain and Bandwidth Enhancement of Microstrip Patch Antenna ArrayChirag Arora1, Shyam S. Pattnaik2, and Rudra N. Baral Progress In Electromagnetics Research B, Vol. 76, 73-85, 2017 